M. Koike

Nagoya Math. J.

Vol. 62 (1976), 29-39

\title{
ON CERTAIN ABELIAN VARIETIES OBTAINED FROM NEW FORMS OF WEIGHT 2 ON $\Gamma_{0}\left(3^{4}\right)$ AND $\Gamma_{0}\left(3^{5}\right)$
}

\author{
MASAO KOIKE
}

Let $N$ be a positive integer and let $\Gamma_{0}(N)$ be the subgroup of $S L(2, Z)$ defined by all matrices $\left(\begin{array}{ll}a & b \\ c & d\end{array}\right)$ with $c \equiv 0(\bmod N)$. Let $S_{2}\left(\Gamma_{0}(N)\right)$ be the space of holomorphic cusp forms of weight 2 with respect to $\Gamma_{0}(N)$ and let $S_{2}^{0}\left(\Gamma_{0}(N)\right)$ be the "essential part" of $S_{2}\left(\Gamma_{0}(N)\right)$, which is defined in [1].

Let $f \in S_{2}^{0}\left(\Gamma_{0}\left(3^{4}\right)\right)$ be a common eigenfunction of all the Hecke operators, which is called a new form on $\Gamma_{0}\left(3^{4}\right)$, and let $A$ be an abelian variety defined over $\boldsymbol{Q}$ obtained from $f$ which is defined by Shimura in [10, Th. 1]. It is known that $A$ is a 2-dimensional, $Q$-simple abelian variety and that there exists an abelian subvariety $B$ of $A$ defined over $Q(\sqrt{-3})$ such that $A$ is isogenous over $Q(\sqrt{-3})$ to $B \times B^{s}$. Here $B^{e}$ is the transform of $B$ by the non-trivial automorphism $\varepsilon$ of $Q(\sqrt{-3})$.

In this paper, we shall consider the field $L$ generated over $Q(\sqrt{-3})$ by the coordinates of 3 -section points of $B$. The obtained result is as follows :

Proposition. $L$ is the field generated by a primitive $3^{2}$-th root of unity over $\boldsymbol{Q}$.

Moreover, we shall prove the following theorem by using the above result:

THEOREM. $B$ is defined up to e-transform by the equation: $y^{2}-3 x y$ $+e^{2 \pi \sqrt{-1 / 3}} y=x^{3}$ and its conductor over $Q(\sqrt{-3})$ is $(\sqrt{-3})^{6}$.

We shall also make the same consideration in the case of $\Gamma_{0}\left(3^{5}\right)$.

The author wishes to express his hearty thanks to Professor K. Miyake and Mr. T. Hadano for their valuable discussions.

Received April 19, 1975.

This work is partially supported by Fujukai. 
Notations. Let $\boldsymbol{Z}, \boldsymbol{Q}, \boldsymbol{R}$ and $\boldsymbol{C}$ be the rational integers, the rational numbers, the real numbers and the complex numbers. Let $k$ denote the field $Q(\sqrt{-3})$.

\section{Preliminaries in the case of $\Gamma_{0}\left(3^{4}\right)$}

Ligozat [6] gave a basis of $S_{2}^{0}\left(\Gamma_{0}\left(3^{4}\right)\right)$ by using the same method as in Honda-Miyawaki's paper [3]. The result is as follows: let $\eta(\tau)$ be Dedekind's $\eta$-function. Put $f_{1}=\eta(9 \tau)^{6} / \eta(3 \tau) \eta(27 \tau)$ and $f_{2}=\eta(3 \tau) \eta(9 \tau)^{2} \eta(27 \tau)$. Then we have $\operatorname{dim}_{C} S_{2}^{0}\left(\Gamma_{0}\left(3^{4}\right)\right)=2$ and $f_{1}$ and $f_{2}$ form a basis of $S_{2}^{0}\left(\Gamma_{0}\left(3^{4}\right)\right)$.

Put $F^{(1)}=f_{1}+\sqrt{3} f_{2}$ and $F^{(2)}=f_{1}-\sqrt{3} f_{2}$. Let $F^{(1)}=\sum_{n \geq 1} a_{n} z^{n}$, $a=e^{2 \pi \sqrt{-1} \tau}$ be the Fourier expansion. It is easily proved that $F^{(1)}$ and $F^{(2)}$ are new forms on $\Gamma_{0}\left(3^{4}\right)$. Let $\chi(n)=\left(\frac{-3}{n}\right)$ be the Legendre symbol. For $f=\sum_{n \geq 1} a(n) z^{n}$, we define $f_{x}=\sum_{n \geq 1} a(n) \chi(n) z^{n}$. Then it is easily proved that $F_{x}^{(1)}=F^{(2)}$ and $F_{x}^{(2)}=F^{(1)}$. Put $K=Q(\sqrt{3}), k=Q(\sqrt{-3})$ and let $\rho, \varepsilon$ be the non-trivial automorphisms of $K, k$ respectively.

By virtue of [10,Th. 1], we obtain an abelian variety $A$ and an isomorphism $\theta$ of $K$ into End $(A) \otimes Q . A$ and $\theta(a)$ for every $\theta(a) \in$ $\theta(K) \cap \operatorname{End}(A)$, are rational over $Q$. Since $\chi$ is the primitive character $\bmod 3$ and it holds $a \equiv d(\bmod 3)$ for every $\left(\begin{array}{ll}a & b \\ c & d\end{array}\right) \in \Gamma_{0}\left(3^{4}\right)$, the condition (4.8) in [10] is satisfied. Since we have $F_{x}^{(1)}=F^{(2)}$, the condition (4.9) in [10] is also satisfied. Therefore, by virtue of [10, Prop. 8 and Prop. 9], there exists an endomorphism $\eta$ of $A$, defined over $k$, such that (i) $\eta^{6}=-\eta$, (ii) $\eta^{2}=-3 \mathrm{id}_{A}$, (iii) $\eta \circ \theta(\alpha)=\theta\left(a^{\rho}\right) \circ \eta$ for every $a \in K$.

Put $B=(\eta-\theta(\sqrt{\mathbf{3}})) A$. Since $(\eta-\theta(\sqrt{3}))^{2}=\eta^{2}+\theta(3)=0, B$ is an elliptic curve defined over $k$ and $A=B+B^{\star}$.

Proposition 1. $\operatorname{End}_{\boldsymbol{Q}}(B)=\boldsymbol{Q}$.

Proof. We consider the $p$-th power Frobenius endomorphism $\varphi_{p}$ of $B \bmod \mathfrak{p}$, where $\mathfrak{p}$ is a prime ideal in $K$ such that $N \mathfrak{p}=p, \chi(p)=1$. Take $p=7,13$. Then we have $a_{7}=2$ and $a_{13}=-1$. Hence one knows $\boldsymbol{Q}\left(\varphi_{7}\right) \cong \boldsymbol{Q}(\sqrt{-6})$ and $\boldsymbol{Q}\left(\varphi_{13}\right) \cong \boldsymbol{Q}(\sqrt{-51})$. This completes the proof by the same argument as that of [8, Th. 7.39].

Q.E.D.

\section{The field generated by the coordinates of 3-section points of $B$}

In the case of $\Gamma_{0}\left(5^{3}\right)$ and $\Gamma_{0}\left(7^{3}\right)$, Doi and Yamauchi [2] studied such 
abelian varieties $B$ and the fields generated by the coordinates of certain points of finite order on $B$. Their idea comes from the work of Shimura [8] in the case of "Neben"-type. On the other hand, in the case of "Neben"-type, Yamauchi [12] studied another type of fields generated by the coordinates of $l$-section points of the same abelian varieties; $l$ is an odd prime number with respect to which Doi has found some arithmetical congruences for the Fourier coefficient of new forms. However, such arithmetical congruences are proved only in the case of prime level (an extended result is proved in [4]).

In the following, we shall show that we have such arithmetical congruences mod 3 for the Fourier coefficients of $F^{(1)}$. Hence, we shall determine the field over $k$ generated by the coordinates of 3 -section points of $B$ by the same argument as in Yamauchi's paper [12].

Proposition 2. Let $F^{(1)}=\sum_{n \geq 1} a_{n} z^{n}$ denote the Fourier expansion. Then it holds

$$
\begin{array}{ll}
1+p-a_{p} \equiv 0(\bmod 3) & \text { if } \chi(p)=1, \\
(1+p)^{2}-a_{p}^{2} \equiv 0(\bmod 3) & \text { if } \chi(p)=-1,
\end{array}
$$

for every prime $p \neq 3$.

Proof. Let $f_{i}=\sum_{n \geq 1} b_{i}(n) z^{n}$, for $i=1,2$, be their Fourier expansions. It is clear $b_{i}(n) \in Z$ for all $n, i=1,2$. For any prime $p \neq 3$, it is obvious we have $a_{p}=b_{1}(p)$ if $\chi(p)=1$ and we have $a_{p}=b_{2}(p) \sqrt{3}$ if $\chi(p)=-1$. Since $\chi(p)=-1$ means $p \equiv 2(\bmod 3)$, we have $(1+p)^{2} \equiv 0$ $(\bmod 3)$ and $a_{p}^{2}=3 b_{2}(p)$, so $(1)$ is proved to be valid if $\chi(p)=-1$. Let $\Delta(\tau)=z \prod_{n \geq 1}\left(1-z^{n}\right)^{24}=\prod_{n \geq 1} \tau(n) z^{n}$ be Ramanujan's function. Then $f_{1} \equiv \Delta(\tau)(\bmod 3)$ holds, since $\left(1-z^{3 n}\right) \equiv\left(1-z^{n}\right)^{3}(\bmod 3)$. Hence we have $a_{p} \equiv \tau(p)(\bmod 3)$ if $\chi(p)=1$. On the other hand, it is well known that, for any prime $p, \tau(p) \equiv 1+p(\bmod 3)$ holds if $p \equiv 1(\bmod 3)$. Therefore (1) is completely proved.

Q.E.D.

Proposition 3. Let $L$ be a field over $Q(\sqrt{-3})$ generated by the coordinates of all 3-section points of $B$. Then $L$ coincides with $Q\left(\zeta_{9}\right)$, where $\zeta_{9}$ is a primitive 9-th root of unity.

Proof. The proof is entirely similar to that of Theorem in [12]. For small prime $p, a_{p}$ is easily calculated by hand and the following is 
a part of them ${ }^{(*)}$ :

$\begin{array}{rrrrccc}p & a_{p} & a_{p}^{2}-4 p & r & \mathfrak{p} & \operatorname{Ind}_{r} \omega & \operatorname{Ind}_{r} \sqrt{-3} \\ 7 & 2 & -24 & 2 & 2-\sqrt{-3} & 4 & 2 \\ 7 & 2 & -24 & 2 & 2+\sqrt{-3} & 2 & 5 \\ 61 & -7 & -195 & 10 & 7-2 \sqrt{-3} & 40 & 36\end{array}$

From Proposition 2 and the above table, it follows easily that $L$ is a Galois extension of $Q(\sqrt{-3})$ of degree 3. In this case, the primitive 3-th root $\omega=e^{2 \pi \sqrt{-1} / 3}$ of unity is already contained in the base field $Q(\sqrt{-3})$. We know that any prime divisor $p$ of $Q(\sqrt{-3})$ is unramified in $L$, if $\mathfrak{p} \neq(\sqrt{-3})$, so we have $L=Q(\sqrt{-3}, \alpha)$, where $\alpha^{3}$ is equal to one of $\omega$, $\sqrt{-3}, \omega \sqrt{-3}, \omega^{2} \sqrt{-3}$. From the above table, we have $\alpha^{3}=\omega$ by the same argument as in [12]. Hence Proposition 3 is completely proved.

Q.E.D.

\section{The defining equation of the elliptic curve $B$}

In this section we shall obtain the defining equation of $B$. From Proposition 3 , it follows that there exists a $k$-rational, proper 3 -section point on $B$. The conductor of $B$ over $k$ is obviously a power of $(\sqrt{-3})$. Since the base field $k$ is imaginary quadratic, the above properties yield a finite number of elliptic curves. The precise result is as follows:

LEMMA $^{(*)}$. Let $E$ be an elliptic curve defined over $k$ such that

(i) the conductor of $E$ over $k$ is a power of $(\sqrt{-3})$,

(ii) there exists a k-rational, proper 3-section point on $E$.

Then the defining equation of $E$ is one of the followings up to $k$ isomorphisms and $\varepsilon$-transform, where $\varepsilon$ is the non-trivial automorphism of $k$ :

$$
\begin{aligned}
& E_{1, \pm}: y^{2} \pm 3 x y+\omega y=x^{3}, \\
& E_{2}: y^{2}-3 x y+\omega \sqrt{-3} y=x^{3}, \\
& E_{3}: y^{2}-3 \sqrt{-3} x y+3 \omega y=x^{3}, \\
& E_{4}: y^{2}+\omega y=x^{3}, \\
& E_{5}: y^{2}+\omega \sqrt{-3} y=x^{3}, \\
& E_{6}: y^{2}+3 \omega y=x^{3},
\end{aligned}
$$

(*) In the table, $r$ denotes a primitive $\operatorname{root} \bmod p, \operatorname{Ind}{ }_{r} \omega$ is the index of $\omega(\bmod \mathfrak{p})$ with respect to $r$.

(*) This lemma is essentially due to Mr. T. Hadano. 


$$
\begin{aligned}
& E_{7}: y^{2}+y=x^{3}, \\
& E_{8}: y^{2}+\sqrt{-3} y=x^{3}, \\
& E_{9}: y^{2}+3 y=x^{3},
\end{aligned}
$$

where $\omega$ is the primitive 3 -th root $e^{2 \pi \sqrt{-1} / 3}$ of unity.

Proof. From (i) and (ii), the defining equation of $E$ is written by $y^{2}+a x y+b y=x^{3}$ with some integers $a, b \in k$ such that the discriminant $\Delta=-b^{3}\left(a^{3}+27 b\right)$ is equal to $\pm \sqrt{-3^{n}}, \pm \omega \sqrt{-3^{n}}$ or $\pm \omega^{2} \sqrt{-3}$. Up to $k$-isomorphisms and $\varepsilon$-transform, we may assume $b$ is one of $\sqrt{-3^{\alpha}}$, $\omega \sqrt{-3}^{\alpha}$ and $\omega^{2} \sqrt{-3}^{\alpha}$ with $\alpha=0,1,2$ and $\Delta$ is $\pm \sqrt{-3}^{n}$ or $\pm \omega^{2} \sqrt{-3}^{n}$. The argument being similar, we deal with only the case $\Delta= \pm \omega^{2} \sqrt{-3}^{n}$. Put $a=\sqrt{-3}^{\beta} c$ with some integer $c \in k$ such that $(c, \sqrt{-3})=1$. Put $c=$ $\frac{1}{2}(x+y \sqrt{-3})$ with $x, y \in Z$. (1) $b=\sqrt{-3}^{\alpha}$. Since we have $\sqrt{-3^{3 \beta}} c^{3}-$ $\sqrt{-3}^{\alpha+6}= \pm \omega^{2} \sqrt{-3}^{n-3 \alpha}$, two of $\{3 \beta, \alpha+6, n-3 \alpha\}$ are equal to each other. If $3 \beta=\alpha+6 \leq n-3 \alpha$, we have $c^{3}-1= \pm \omega^{2} \sqrt{-3}^{n-4 \alpha-6}$. Put $\nu=$

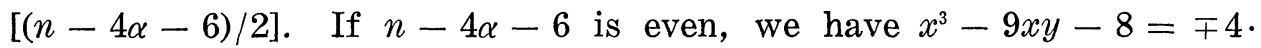
$(-3)^{\nu}$ and $3 x^{2} y-3 y^{3}-8=\mp 4 \cdot(-3)^{\nu}$. If $n-4 \alpha-6$ is odd, we have $x^{3}-9 x y^{2}-8=\mp 4(-3)^{\nu+1}$ and $3 x^{2} y-3 y^{2}-8=\mp 4(-3)^{\nu}$. It is obvious that these equations have no integral solutions $x$ and $y$. In other two cases, it is similarly proved that there is no integral solution $c$. (2) $b=\omega \sqrt{-3}^{\alpha}$. We have $\sqrt{-3^{3 \beta}} c^{3}-\omega \sqrt{-3^{\alpha+6}}= \pm \omega^{2} \sqrt{-3^{n-3 \alpha}}$. (i) $3 \beta=\alpha+6$ $\leq n-3 \alpha$. Hence if $n-4 \alpha-6$ is even, we have $x^{3}-9 x y^{2}+4=\mp 4(-3)^{\nu}$ and $3 x^{2} y-3 y^{3}-4=\mp 4(-3)^{\nu}$, and if $n-4 \alpha-6$ is odd, we have $x^{3}-$ $9 x y^{2}+4=4(-3)^{\nu+1}$ and $3 x^{2} y-3 y^{3}-4=\mp 4(-3)^{\nu}$. Then it is clear $\nu=0$. In the case $n-4 \alpha-6=0$, the solutions are $\alpha=0, \beta=2$ and $c=-1,-\omega,-\omega^{2}$. In the case $n-4 \alpha-6=1$, the solutions are $\alpha=0$, $\beta=2$ and $c=1, \omega, \omega^{2}$. Thus $E_{1, \pm}$ are obtained. (ii) $3 \beta=n-3 \alpha<\alpha+6$. We have $c^{3} \pm \omega^{2}=\omega \sqrt{-3^{4 \alpha+6-n}}$. Put $\nu^{\prime}=[(4 \alpha+6-n) / 2]$. Then, if $4 \alpha+6-n$ is even, we have $x^{3}-9 x y^{2} \mp 4=-4(-3)^{\nu^{\prime}}$ and $3 x^{2} y-3 y^{3} \mp 4$ $=4(-3)^{\nu^{\prime}}$ and, if $4 \alpha+6-n$ is odd, we have $x^{3}-9 x y^{2} \mp 4=4(-3)^{\nu^{\prime}+1}$ and $3 x^{2} y-3 y^{3} \mp 4=-4(-3)^{\nu^{\prime}}$. It is obvious $\nu^{\prime}=0$, so we have $4 \alpha+$ $6-n=1$. Hence the solutions are $\alpha=1, \beta=2$ and $c=-1,-\omega,-\omega^{2}$. Thus $E_{2}$ is obtained. (iii) $n-3 \alpha=\alpha+6<3 \beta$. We have $\omega \pm \omega^{2}=$ $c^{3} \sqrt{-3^{3 \beta-\alpha-6}}$. Hence the solutions are $\alpha=2, \beta=3$ and $c=1, \omega, \omega^{2}$. Thus $E_{3}$ is obtained. (3) $b=\omega^{2} \sqrt{-3^{\alpha}}$. By the same argument as above, the solutions are $c=0$ and $\alpha=0,1,2$. Thus $E_{4}, E_{5}$ and $E_{6}$ are obtained. 
$E_{7}, E_{8}$ and $E_{9}$ are obtained from the case $\Delta= \pm \sqrt{-3^{n}}$.

Q.E.D.

THEOREM 1. The defining equation of $B$ is given by

$$
y^{2}-3 x y+\omega y=x^{3}
$$

up to e-transform and its conductor over $k$ is $(\sqrt{-3})^{6}$.

Proof. Since $B$ satisfies the conditions (i) and (ii) in Lemma, its defining equation is given by one of the above table. On the other hand, $L$-function of the elliptic curve $B$ over $k$ is equal to the Euler product

$$
\prod_{p \neq 3}\left(1-a_{p} p^{-s}+p^{1-2 s}\right)^{-1}\left(1-a_{p}^{\rho} p^{-s}+p^{1-2 s}\right)^{-1}
$$

up to 3-factor, where $a_{p}$ is the $p$-th Fourier coefficient of $F^{(1)}$. For prime $p \neq 3$, with $\chi(p)=1$, we may put $p=\mathfrak{p p}^{\prime}$ with prime ideals $\mathfrak{p} \neq \mathfrak{p}^{\prime}$ in $k$. Since $a_{p}=a_{p}^{\rho}$ holds if $\chi(p)=1$, the local $\mathfrak{p}$ - and $\mathfrak{p}^{\prime}$-factor of $L$ function of $B$ coincide to each other. The following is the table of the number of $F_{p}$-rational points of $E \bmod \mathfrak{p}$ with $N \mathfrak{p}=p$ :

$\begin{array}{lrrrrrr} & 2-\sqrt{-3} & 2+\sqrt{-3} & 1-2 \sqrt{-3} & 1+2 \sqrt{-3} & 4-\sqrt{-3} & 4+\sqrt{-3} \\ E_{1,+} & 12 & 9 & 12 & 18 & 18 & 27 \\ E_{1,-} & 6 & 6 & 15 & 15 & 18 & 18 \\ E_{2} & 9 & 12 & 18 & 12 & 27 & 18 \\ E_{3} & 9 & 9 & 9 & 9 & 21 & 21 \\ E_{4} & 3 & 12 & 12 & 21 & 27 & 27 \\ E_{5} & 9 & 3 & 9 & 12 & 21 & 21 \\ E_{6} & 12 & 9 & 21 & 9 & 12 & 12 \\ E_{7} & 9 & 9 & 9 & 9 & 27 & 27 \\ E_{8} & 12 & 12 & 21 & 21 & 21 & 21 \\ E_{9} & 3 & 3 & 12 & 12 & * & * \\ B & 6 & 6 & 15 & 15 & 18 & 18 \\ B_{1} & 9 & 9 & 9 & 9 & 21 & 21\end{array}$

Hence the defining equation of $B$, up to $\varepsilon$-transform, is given by $y^{2}-$ $3 x y+\omega y=x^{3}$. The conductor of $B$ is easily calculated from this equation.

Q.E.D.

COROLLARY. L-function of $B$ over $k$ is equal to

$$
\prod_{p}\left(1-a_{p} p^{-s}+p^{1-2 s}\right)^{-1}\left(1-a_{p}^{\rho} p^{-s}+p^{1-2 s}\right)^{-1}
$$


where $a_{p}$ is the p-th Fourier coefficient of $F^{(1)}$.

Proof. The reduction $\bmod (\sqrt{-3})$ of the minimal model of $B$ is proved to be of additive type from Theorem 1 , so the local $(\sqrt{-3})$-factor of $L$-function is equal to 1 .

Q.E.D.

\section{Preliminaries in the case of $\boldsymbol{\Gamma}_{0}\left(3^{5}\right)$}

Ligozat [6] also gave a basis of $S_{2}^{0}\left(\Gamma_{0}\left(3^{5}\right)\right)$. The result is as follows: put $g_{1}=\eta(9 \tau)^{3} \eta(27 \tau)^{3} / \eta(3 \tau) \eta(81 \tau), g_{2}=\eta(9 \tau)^{4} \eta(81 \tau)^{2} / \eta(3 \tau) \eta(27 \tau), g_{3}=\eta(3 \tau)^{2} \eta$ $(81 \tau)^{2}, \quad g_{4}=\eta(3 \tau)^{2} \eta(27 \tau)^{4} / \eta(9 \tau) \eta(81 \tau), \quad g_{5}=\eta(3 \tau) \eta(9 \tau) \eta(27 \tau) \eta(81 \tau), g_{7}=\eta(9 \tau)^{5} \eta$ $(81 \tau) / \eta(3 \tau) \eta(27 \tau), \quad g_{8}=\eta(3 \tau)^{2} \eta(9 \tau) \eta(81 \tau), \quad g_{9}=\eta(3 \tau) \eta(27 \tau)^{5} / \eta(9 \tau) \eta(81 \tau), \quad g_{10}=$ $\eta(3 \tau) \eta(27 \tau) \eta(81 \tau)^{2}$. Let $T(2)$ be the Hecke operator acting on $S_{2}^{0}\left(\Gamma_{0}\left(3^{5}\right)\right)$ as a linear endomorphism which is defined by $f(\tau) \rightarrow f(\tau) \mid T(2)=f\left(\frac{\tau}{2}\right)+$ $f\left(\frac{\tau+1}{2}\right)+2 f(2 \tau) . \quad$ Put $g_{6}=g_{5}\left|T(2), g_{11}=g_{7}\right| T(2)$ and $g_{12}=g_{9} \mid T(2)$.

Then $\left\{g_{i}\right\}, 1 \leq i \leq 12$, are linearly independent over $C$ and form a basis of $S_{2}^{0}\left(\Gamma_{0}\left(3^{5}\right)\right)$. He also calculated the eigenvalues of $T(2) ;\{0,0, \pm \sqrt{\mathbf{3}}$, $\left.\pm \sqrt{6}, \pm \sqrt{3+\alpha_{i}}, 1 \leq i \leq 3\right\}$ are all the eigenvalues of $T(2)$, where $\alpha_{i}$, $1 \leq i \leq 3$, are three roots of the equation $X^{3}-9 X-9=0$.

From this, we can construct new forms on $\Gamma_{0}\left(3^{5}\right)$. We are interested in only new forms from which 2 -dimensional, $Q$-simple abelian varieties are obtained. Such new forms are as follows: put $G^{(1)}=g_{1}-3 g_{3}+$ $\sqrt{3}\left(2 g_{5}+\frac{1}{3} g_{6}\right), \quad G^{(3)}=g_{1}-3 g_{3}-\sqrt{3}\left(2 g_{5}+\frac{1}{3} g_{6}\right), \quad G^{(2)}=2 g_{1}-3 g_{2}-g_{4}$ $+\sqrt{6}\left(-g_{5}+\frac{1}{3} g_{6}\right)$ and $G^{(4)}=2 g_{1}-3 g_{2}-g_{4}-\sqrt{6}\left(-g_{5}+\frac{1}{3} g_{6}\right)$. Then we have $G^{(1)}\left|T(2)=\sqrt{3} G^{(1)}, G^{(3)}\right| T(2)=-\sqrt{3} G^{(3)}, G^{(2)} \mid T(2)=\sqrt{6} G^{(2)}$ and $G^{(4)} \mid T(2)=-\sqrt{6} G^{(4)}$. Hence $G^{(i)}, 1 \leq i \leq 4$, are new forms on $\Gamma_{0}\left(3^{5}\right)$. We have also $G_{\chi}^{(1)}=G^{(3)}, G_{x}^{(3)}=G^{(1)}, G_{\chi}^{(2)}=G^{(4)}$ and $G_{\chi}^{(4)}=G^{(2)}$, where $\chi(n)=\left(\frac{-3}{n}\right)$ is the primitive character $\bmod 3$. Let $G^{(i)}=\sum_{n \geq 1} a_{i}(n) z^{n}$, for $i=1,2$ denote their Fourier expansions.

Put $K_{1}=Q(\sqrt{3})$ and $K_{2}=Q(\sqrt{6})$. For each $i=1,2$, let $\rho_{i}$ be the non-trivial automorphism of $K_{i}$. For each $i=1,2$, let $\left(A_{i}, \theta_{i}\right)$ be an abelian variety and an isomorphism of $K_{i}$ into $\operatorname{End}_{Q}\left(A_{i}\right)$ which are obtained from $G^{(i)}$ by virtue of [10,Th. 1]. Then, by the same reason as in $\S 1$, there exists an endomorphism $\eta_{1}\left(\right.$ resp. $\left.\eta_{2}\right)$ of $A_{1}$ (resp. $A_{2}$ ), defined 
over $k$, such that (i) $\eta_{i}^{e}=-\eta_{i}$, (ii) $\eta_{i}^{2}=-3 \mathrm{id}_{A_{i}}$, (iii) $\eta_{i} \circ \theta_{i}(a)=\theta_{i}\left(\alpha^{\rho_{i}}\right) \circ \eta_{i}$, for every $a \in K_{i}$.

Put $B_{1}=\left(\eta_{1}-\theta_{1}(\sqrt{3})\right) A . \quad B_{1}$ is an elliptic curve defined over $k$ such that $A_{1}=B_{1}+B_{1}^{e}$, by the same argument as in $\S 1$.

Proposition 4. $\operatorname{End}_{Q}\left(B_{1}\right)=Q$.

Proof. Since we have $a_{1}(7)=-1, a_{1}(31)=5$, this completes the proof by the same argument as that of Proposition 1.

Q.E.D.

As for $A_{2}$, we have the following:

Proposition 5. $A_{2}$ is a simple abelian variety and $\operatorname{End}_{Q}\left(A_{2}\right)$ coincides with a division quaternion algebra $Q\left[\theta_{2}(1), \theta_{2}(\sqrt{6}), \eta_{2}, \eta_{2} \circ \theta_{2}(\sqrt{6})\right]$ over $\boldsymbol{Q}$.

Proof. Put $D=Q\left[\theta_{2}(1), \theta_{2}(\sqrt{6}), \eta_{2}, \eta_{2} \circ \theta_{2}(\sqrt{6})\right]$. We shall show $D$ is a division algebra. Since $D \otimes_{Q} R \cong M_{2}(R)$ and $\eta_{2} \circ \theta_{2}(\sqrt{6})=\theta_{2}(-\sqrt{6}) \circ \eta_{2}$, there exists a $Q$-isomorphism $\iota: D \rightarrow M_{2}(R)$ such that $\iota\left(\theta_{2}(\alpha+b \sqrt{6})\right)=$ $\left(\begin{array}{cc}a+b \sqrt{6}, & 0 \\ 0, & a-b \sqrt{6}\end{array}\right)$ with $a, b \in \boldsymbol{Q}$ and $\iota\left(\eta_{2}\right)=\left(\begin{array}{ll}0 & \beta \\ \gamma & 0\end{array}\right)$ with $\beta \gamma=-3$. If $\theta_{2}(a+b \sqrt{6})-\eta_{2}$ is a zero divisor of $D$, we have $\operatorname{det}\left(\begin{array}{cc}a+b \sqrt{6}, & -\beta \\ -\gamma, & a-b \sqrt{6}\end{array}\right)$ $=(a+b \sqrt{6})(a-b \sqrt{6})+3=0$. One knows the norm of the fundamental unit of $Q(\sqrt{6})$ is 1 and $(3-\sqrt{6})(3+\sqrt{6})=3$, so there is no solution $a, b \in Q$ such that $(a+b \sqrt{6})(a-b \sqrt{6})+3=0$. Hence $D$ is proved to be a division algebra. We suppose $A_{2}$ is not simple. Then $A_{2}$ is isogenous to $B \times B^{\prime}$ with some elliptic curves $B, B^{\prime}$. If $B$ is not isogenous to $B^{\prime}$, it follows $\operatorname{End}_{Q} A_{2} \cong \operatorname{End}_{Q} B \oplus \operatorname{End}_{Q} B^{\prime}$. Since $\operatorname{End}_{Q} A_{2}$ $\supset D$, this is a contradiction. We may assume $B$ is isogenous to $B^{\prime}$. Then we have $\operatorname{End}_{Q} A_{2} \cong M_{2}\left(\operatorname{End}_{Q} B\right)$. If $B$ is not of $C M$-type, we have $\operatorname{End}_{Q} A_{2} \cong M_{2}(Q):$ this is a contradiction. If $B$ is of $C M$-type, the Mellin transform of $G^{(2)}$ is the $L$-function with a primitive Grössencharactere of certain imaginary quadratic field by virtue of [9, Prop. 1.6]. From Proposition 6, it follows that $a_{2}(p)$ is not equal to zero if $p \equiv 1(\bmod 3)$. We have $a_{2}(5) \neq 0$; this is a contradiction. Hence $A_{2}$ is proved to be simple. Therefore $\operatorname{End}_{\boldsymbol{Q}} A_{2}$ is a division algebra over $\boldsymbol{Q}$ with a positive involution. All algebra of this type are classified in [7, Prop. 1]. Since $\operatorname{End}_{Q} A_{2} \supset D, \operatorname{End}_{Q} A_{2}$ is not of type I. Let $F$ be the center of $\operatorname{End}_{Q} A_{2}$. Suppose $F \neq Q$. Put $d=[F: Q]$. Then we have $d \mid 4$ by [11, Prop. $1, \S 5-$ 
1]. If $d=4$, we have $\operatorname{End}_{Q} A_{2}=F$ : this is a contradiction. $\theta_{2}(\sqrt{6})$ and $\eta_{2}$ are not contained in $F$. Let $D_{1}$ (resp. $D_{2}$ ) be the subalgebra over $F$ generated by $\theta_{2}(\sqrt{6})$ (resp. $\left.\eta_{2}\right)$. If $d=2$, either $D_{1}$ or $D_{2}$ is a field of degree 4 over $Q$. Then, by [11, Prop. 6, §5-1], $\operatorname{End}_{Q} A_{2}$ is proved to be a field: this is a contradiction. Hence we have $F=Q$. Since $\operatorname{End}_{Q} A_{2}$ $\supset D, \operatorname{End}_{Q} A_{2}$ is of type II and coincides with $D$.

Q.E.D.

\section{Arithmetical congruences for $G^{(1)}$ and $G^{(2)}$ and the field generated by the coordinates of 3-section points of $B_{1}$}

We shall make the same consideration on $B_{1}$ as in $\S 2$.

Proposition 6. Let $G^{(i)}=\sum_{n \geq 1} a_{i}(n) z^{n}, i=1,2$, be the above new forms on $\Gamma_{0}\left(3^{5}\right)$. Let $\mathfrak{p}_{i}, i=1,2$, be the prime divisor of $K_{i}$ lying on 3 of $\boldsymbol{Q}$. Then it holds $1+p-a_{i}(p) \equiv 0\left(\bmod \mathfrak{p}_{i}\right)$ for every prime $p \neq 3$, $i=1,2$.

The proof is entirely similar to that of Proposition 2, so we omit the proof.

Proposition 7. Let $L_{1}$ be the field over $k$ generated by the coordinates of 3-section points of $B_{1}$. Then Gal $\left(L_{1} / k\right)$ is isomorphic to the cyclic group of order 3 and $L_{1}$ coincides with either $k(\sqrt[3]{\omega \sqrt{-3}})$ or $k\left(\sqrt[3]{\omega^{2} \sqrt{-3}}\right)$.

Proof. The following is the table of $a_{1}(p)$ for certain primes $p$ :

$\begin{array}{rrrrccc}p & a_{p} & a_{p}^{2}-4 p & r & \mathfrak{p} & \operatorname{Ind}_{r} \omega & \operatorname{Ind}_{r} \sqrt{-3} \\ 19 & -1 & -75 & 10 & 4-\sqrt{3} & 6 & 16 \\ 61 & 2 & -240 & 10 & 7-2 \sqrt{3} & 40 & 36\end{array}$

From this table, Proposition 7 is proved to be valid by the same argument as in the proof of Proposition 3.

Q.E.D.

Remark. We can also consider the field $L_{2}$ over $Q$ generated by the coordinates of $\mathfrak{p}_{2}$-section points of $A_{2}$. By using Proposition 6 and by the same argument as in Proposition $3, L_{2} / \boldsymbol{Q}$ is a Galois extension of either degree 6 or degree 2 and $\sqrt{-3}$ belongs to $L_{2}$. However we can not decide its degree over $\boldsymbol{Q}$, because we can not apply Shimura's criterion in this case; for any prime $p$ with $p \equiv 1(\bmod 3), a_{2}(p)$ is a rational integer. Since $K_{2} / Q$ is ramified at 3 , we have $a_{2}(p)^{2}-4 p \equiv 0$ $\left(\bmod \mathfrak{p}_{2}^{2}\right)$. 


\section{The defining equation of the elliptic curve $B_{1}$}

From Proposition 7 , it follows that there exists a $k$-rational, proper 3-section point on $B_{1}$. Hence the defining equation of $B_{2}$ is determined by the same argument as in Theorem 1 . We have already calculated the number of $F_{p}$-rational points of $B_{1}(\bmod \mathfrak{p})$ for small primes there, so the result is as follows:

THEOREM 2. The defining equation of $B_{1}$ is given by

$$
y^{2}-3 \sqrt{-3} x y+3 \omega y=x^{3}
$$

up to e-transform and its conductor over $k$ is $(\sqrt{-3})^{8}$.

\section{Concluding remarks}

Let $\alpha$ be a solution of the equation $X^{3}-3 X^{2}+3=0$. Then $Q(\alpha)$ is the maximal real subfield of the field generated by the primitive $3^{2}$-th root of unity over $Q$. Put $G=(\alpha-1) g_{7}+\left(\alpha^{2}-\alpha-2\right) g_{8}+(\alpha-1) g_{9}+$ $\left(3 \alpha^{2}-3 \alpha-6\right) g_{10}+g_{11}+g_{12}$. Then it is proved that $G$ is a new form on $\Gamma_{0}\left(3^{5}\right)$. Let $\left(A_{3}, \theta_{3}\right)$ be a 3-dimensional abelian variety $A_{3}$ defiñed over $\boldsymbol{Q}$ and an isomorphism $\theta_{3}$ of $Q(\alpha)$ into $\operatorname{End}_{Q} A_{3}$ obtained from $G$. It is proved that $\left(A_{3}, \theta_{3}\right)$ is of principal type. As for $G$, we can prove there exist arithmetical congruences; let $\mathfrak{p}$ be the prime divisor of $\boldsymbol{Q}(\alpha)$ lying on 3 of $\boldsymbol{Q}$. Let $G=\sum_{n \geq 1} b(n) z^{n}$ denote the Fourier expansion. Then it holds $1+p-b(p) \equiv 0(\bmod \mathfrak{p})$ for any prime $p \neq 3$. Moreover it is proved that $b(p)^{2}-4(p) \equiv 0\left(\bmod p^{2}\right)$ for every prime $p$ with $p \equiv 1(\bmod 3)$. Hence, in this case, there exists the same difficulty as in Remark in determining the field generated by the coordinates of $p$-section points of the above abelian variety associated with $G$.

\section{REFERENCES}

[1] A. O. L. Atkin and J. Lehner: Hecke operators on $\Gamma_{0}(m)$, Math. Ann., 185 (1970), 134-160.

[2] K. Doi, and M. Yamauchi: On the Hecke operators for $\Gamma_{0}(N)$ and class fields over real quadratic number fields, J. Math. Soc. Japan, 25 (1973), 629-643.

[ 3 ] T. Honda and I. Miyawaki: Zeta-functions of elliptic curves of 2-power conductor, J. Math. Soc. Japan, 26 (1974), 362-373.

[4] M. Koike: On the congruences between Eisenstein series and cusp forms, in preparation.

[5] O. Kolberg: Congruences for Ramanujan's function $\tau(n)$, Univ. Bergen Årbok Naturvit Rekke 1962.

[6] G. Ligozat: A letter to Mr. T. Hadano. 
[ 7 ] G. Shimura: On analytic families of polarized abelian varieties and automorphic functions, Ann. of Math., 78 (1963), 149-192.

[8] G. Shimura: Introduction to the arithmetic theory of automorphic functions, Publ. Math. Soc. Japan, No. 11 Iwanami Shoten and Princeton University Press, 1971.

[9] G. Shimura: Class fields over real quadratic fields and Hecke operators, Ann. Of Math., 95 (1972), 130-190.

[10] G. Shimura: On the factors of the jacobian variety of a modular function field, J. Math. Soc. Japan, 25 (1973), 523-544.

[11] G. Shimura and Y. Taniyama: Complex multiplication of abelian varieties and its applications to number theory, Publ. Math. Soc. Japan, No. 6, 1961.

[12] M. Yamauchi: On the fields generated by certain points of finite order on Shimura's elliptic curve, J. Math. Kyoto Univ., 14 (1974), 243-255.

Nagoya University 